\title{
le phicomètre : essai de cisaillement direct in situ
}

\author{
the phicometer : in situ direct shear test
}

\author{
G. PHILIPPONNAT \\ Directeur technique de SOPENA S.A.*
}

\section{Résumé}

L'article décrit un nouvel appareil : le phicomètre.

Cet appareil permet de mesurer in situ les caractéristiques mécaniques $\phi$ et C des sols par cisaillement rectiligne.

L'appareil comporte une sonde gonflable munie de coquilles métalliques. Cette sonde est introduite dans un forage de petit diamètre (60 à $64 \mathrm{~mm}$ ). Les coquilles présentent des indentations quî pénètrent dans le terrain sous la pression latérale. On mesure la résistance au cisaillement du sol en exerçant un effort d'arrachement vertical progressif.

Des paliers successifs sont réalisés avec des pressions latérales croissantes.

Actuellement, l'essai est du type non consolidé rapide. Outre la description de l'appareil et du mode opératoire, sont présentés les résultats comparatifs avec des essais de cisaillement rectiligne en laboratoire sur une argile, un sable argileux et un sable pur.

Les résultats obtenus sur un remblai 0/150 mm dont la granulométrie interdit tout prélèvement intact sont également décrits.

Les avantages de cet appareil sont d'une part la possibilité de réaliser des essais sur des sols non ou difficilement prélevables et d'autre part le faible coût de ces essais.

\section{Abstract}

This article describes a new apparatus : the phicometer.

This apparatus permits to measure the mechanical caracteristics of soils - $\varphi$ and C - by in situ direct shear test.

The apparatus includes an inflatable probe equipped with metallic shells. The probe is inserted in a boring of small diameter $(60$ to $64 \mathrm{~mm})$. The shells present indentations which penetrate the soil under lateral pressure. The soil shear strength is measured by application of a progressive vertical traction.

Successive bearings are realized with increasing lateral pressures.

At the moment the test is of the quick unconsolided type. In addition to the description of the instrument and the way to use it, the comparative results with direct shear test in laboratory on clay, clayey sand and pure sand are described.

The results obtained on a fill $0 / 150 \mathrm{~mm}$ whose grain size distribution forbids any undisturbed sample are also described.

The advantages of this apparatus are the possibility to realize tests on soils where undisturbed samples can't be taken and the very low cost of tests.

\footnotetext{
* 5, rue Vincent-Chevard, B.P. 169, 28003 CHARTRES CEDEX.
} 


\section{INTRODUCTION}

L'angle de frottement interne et la cohésion des sols sont deux paramètres fondamentaux pour le géomécanicien.

A l'exception de la cohésion non drainée des argiles molles, ces paramètres ne peuvent être actuellement mesurés directement et d'une façon courante qu'en laboratoire (C.P. WROTH 1984).

On peut estimer que la majorité des sols et des roches altérées ne peut faire l'objet d'essais représentatifs en laboratoire du fait que le sol est imprélevable ou trop grossier ou encore trop sensible au remaniement pendant les manipulations en laboratoire. C'est dire combien le géomécanicien est souvent obligé de se limiter à une simple estimation de ces caractéristiques essentielles.

Différentes tentatives ont été faites dans le passé pour mesurer l'angle de frottement interne et la cohésion in situ.

Il convient de citer notamment :

- le Rhéotest du Professeur BIAREZ (J. COSTE et G. SANGLERAT, 1975),

- le Pressioscissomètre MAZIER (G. MAZIER, 1971),

- le Vane-test canadien (G. FILLIAT, 1981).

Ces trois appareils sont basés sur le même principe inspiré du scissomètre, à savoir :

- dilatation d'ailettes verticales dans le sol à l'aide d'une cellule gonflable sous pression contrôlée, pression qui correspond à la contrainte normale à la surface de cisaillement,

- application de l'effort de cisaillement par rotation des ailettes autour d'un axe vertical.

D'après les publications référencées ci-dessus et les dires de personnes ayant travaillé sur les deux premiers appareils cités, les résultats obtenus avec ces appareils étaient techniquement très intéressants. Malheureusement leur emploi est resté confidentiel.

\section{PRINCIPES GÉNÉRAUX}

Pour la mise au point de l'appareil, nous nous sommes attachés à respecter les principes suivants :

1. Réaliser un essai simple et peu coûteux.

2. Construire un appareil robuste et purement mécanique.

3. Effectuer les essais dans un forage de même diamètre que les essais pressiométriques.

4. Mettre au point un mode opératoire précis et facilement assimilable.
5. Réaliser un grand nombre d'essais dans le cadre d'une recherche expérimentale portant :

a) sur des essais comparatifs avec des essais de laboratoire,

b) sur des essais sur des sols imprélevables,

Cette recherche expérimentale est en cours grâce à l'aide financière de l'A.N.V.A.R. et grâce également à des maîtres d'ouvrages qui ont bien voulu s'intéresser à cet essai. Parmi ces derniers, nous citerons notamment le Centre d'Études des Réseaux des Transports de l'E.D.F. et la S.N.C.F.

\section{PRINCIPE DU PHICOMÈTRE}

L'appareil a été appelé phicomètre pour rappeler qu'il mesure l'angle de frottement interne $\varphi$ et la cohésion des sols in situ.

Actuellement les essais sont du type non-consolidérapide. Afin de bien identifier la façon dont les caractéristiques de cisaillement sont mesurées on leur attribue les symboles $\varphi$ i et ci.

\section{PRINCIPE DE L'ESSAI}

Le principe de l'essai est représenté sur la figure 1.

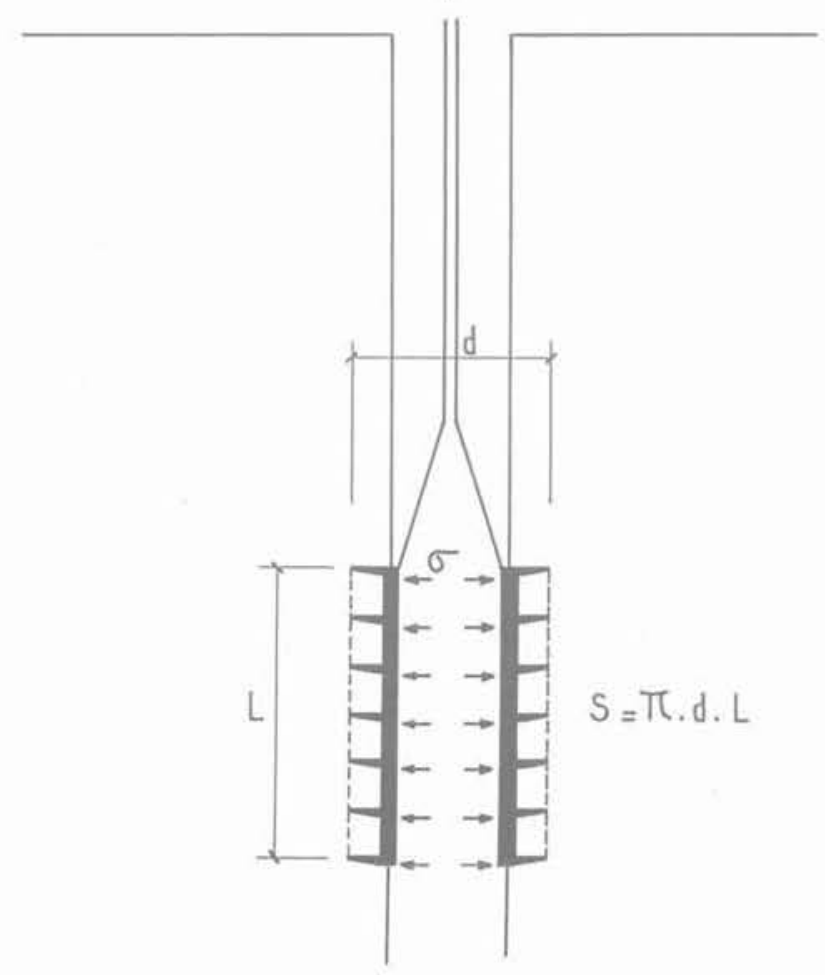

Fig. 1. - Principe de l'essai. 
On commence par exercer sur des coquilles métalliques rigides munies d'indentations horizontales une pression normale $\sigma$.

Les coquilles sont conçues pour pouvoir se dilater afin que les dents pénètrent dans le sol environnant.

La contrainte $\sigma$ s'exerce donc sur la surface circonscrite $\mathrm{S}$, telle que :

$$
\mathrm{S}=\pi \cdot \mathrm{d} \cdot 1
$$

avec :

$\mathrm{d}=$ diamètre extérieur des dents.

1 = longueur de la sonde de mesure.

Ensuite un effort d'arrachement à vitesse contrôlée est appliqué à partir de la surface du sol. L'effort limite mobilisable $T$ sous la contrainte $\sigma$ donne la contrainte de cisaillement correspondante :

$$
\tau=\mathrm{T} / \mathrm{S}
$$

On relève ainsi un certain nombre de couples : $\left\{\tau_{i}, \sigma_{1}\right\}$ par paliers de pressions croissantes successifs.

G. MAZIER (1971) a montré qu'avec une sonde dilatable, on pouvait opérer par paliers successifs au même emplacement. Du fait de la dilatation on cisaille à chaque fois une nouvelle surface. Cette possibilité présente un avantage considérable tant pour la simplicité de l'essai que pour l'homogénéité des résultats.

\section{DESCRIPTION DE L'APPAREIL (figure 2)}

L'appareil comprend 3 organes :

- la sonde phicométrique,

- un organe de liaison.

- un appareillage de surface.

La sonde phicométrique (figure 3) est constituée par des coquilles métalliques de $230 \mathrm{~mm}$ de longueur et $58 \mathrm{~mm}$ de diamètre extérieur initial. La surface de frottement est variable pendant l'essai mais reste voisine de $500 \mathrm{~cm}^{2}$.

Le système de rappel est constitué par des lames d'acier comme dans un tube lanterné. A l'intérieur des coquilles est située une sonde gonflable monocellulaire.

L'organe de liaison comprend :

- des tiges type wagon-drill de 1 pouce $1 / 4$,

- une tubulure reliant la sonde gonflable à l'appareillage de surface.

L'appareillage de surface comprend :

1. Un contrôleur pression-volume permettant de connaître le volume de la sonde et la pression appliquée aux coquilles.

2. Une plaque d'appui sur le sol.

\section{SCHEMA DU PHICOMETRE}

(C) Appareillage de surface

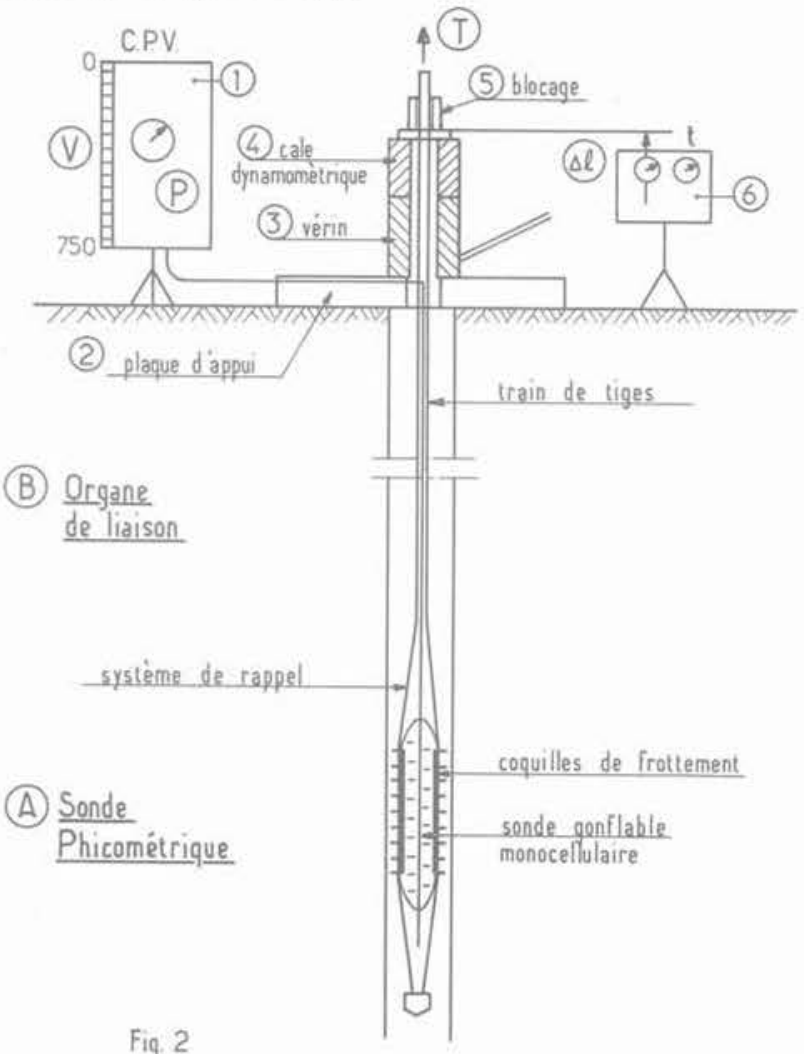

Fig. 2. - Schéma du phicomètre.

3. Un vérin creux permettant d'exercer l'effort d'arrachement.

4. Une cale dynamométrique permettant de mesurer l'effort T d'arrachement.

5. Un système de blocage de l'ensemble.

6. Un cadencemètre constitué lui-même d'un comparateur et d'un chronomètre gradué en 30 secondes pour un tour.

Pendant la phase de cisaillement, le vérin est actionné de façon à conserver les deux aiguilles du chronomètre et du cadencemètre parallèles. De cette façon, la vitesse de cisaillement est constante et de $2 \mathrm{~mm}$ par minute.

La figure 4 montre le dispositif de surface.

La mise au point mécanique du phicomètre a été réalisée par la Société A.P.A.G.E.O.*

- A.P.A.G.E.O, - 59, rue de Rambouillet, 78460 CHEVREUSE. 


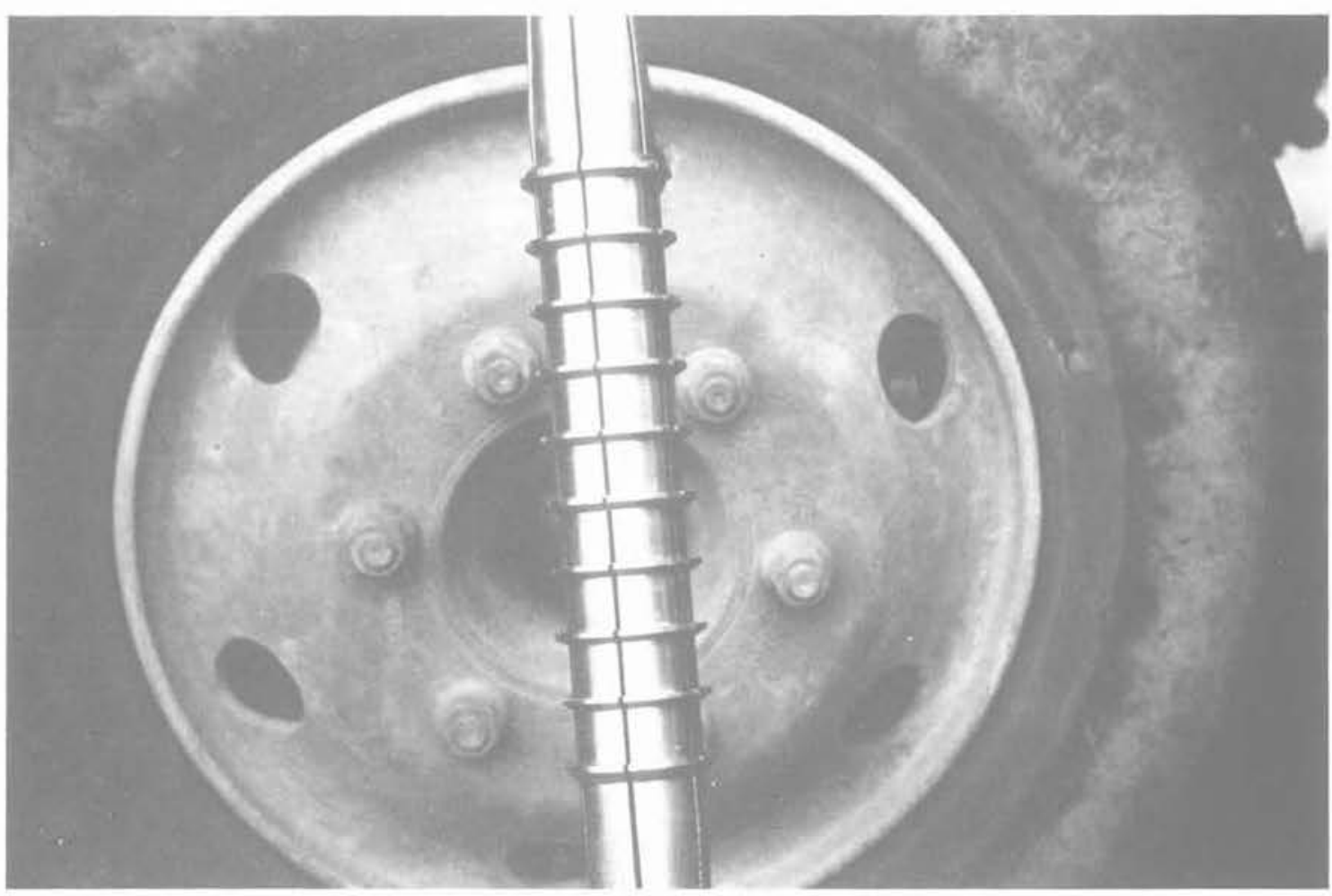

Fig. 3. - Phicomètre. Vue des coquilles dilatables.

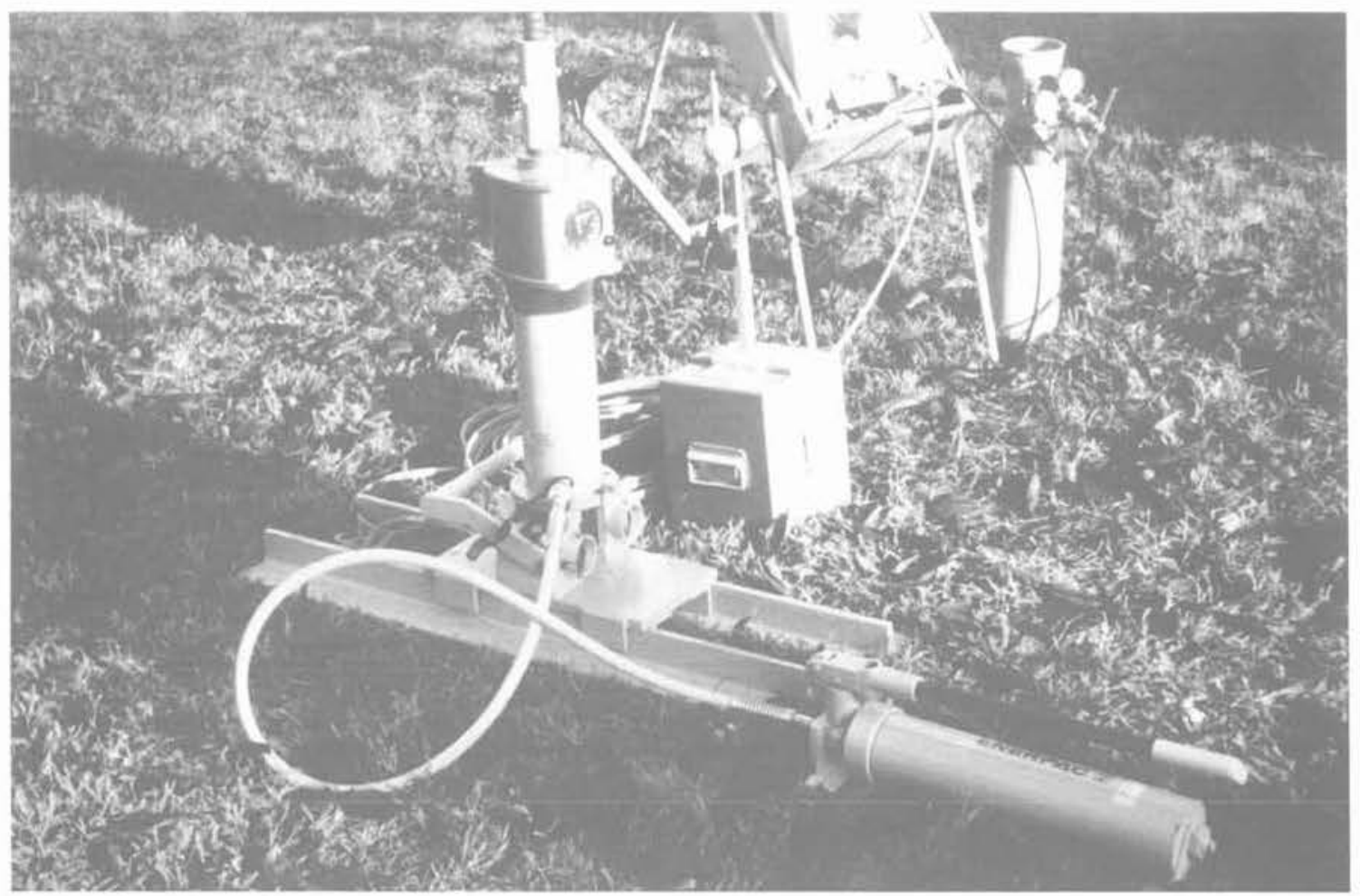

Fig. 4. - Phicomètre. Vue d'ensemble de l'appareillage de surface. 


\section{MODE OPÉRATOIRE}

Un mode opératoire extrêmement détaillé et précis a été établi afin d'assurer la bonne réalisation des essais et leur fidélité. Nous ne décrirons pas en détail ce mode opératoire mais nous allons présenter les phases successives du déroulement des essaís.

\section{Phase 1 : étalonnage :}

Ayant disposé la sonde à l'air libre à côté du sondage, celle-ci est mise en pression en mesurant par paliers la pression nécessaire pour la dilater ainsi que le diamètre externe correspondant. On obtient donc deux courbes (figure 5).

$$
\text { pe }=f(v) \text { et } d=f(v)
$$

avec pe $=$ pression d'étalonnage .

La première courbe permet d'apporter les corrections nécessaires à la mesure de la pression normale pour tenir compte de la raideur de la sonde; la seconde courbe permet de connaître à chaque instant la surface $S$ de contact.
Phase 2 : mise en contact des coquilles et pénétration des dents :

Dans cette phase encore préliminaire, le contact est réalisé par incréments de pression successifs en mesurant sous chaque palier les volumes de la sonde au bout de $15 \mathrm{~s}, 30 \mathrm{~s}$ et $1 \mathrm{mn}$ comme dans l'essai pressiométrique.

La figure 6 représente une feuille d'essai. Dans la partie supérieure de cette feuille intitulée $\propto 1$ mise en contact $x$, sont portées dans la colonne pression, la pression lue au manomètre du contrôleur pressionvolume $\mathrm{pM}$ exprimée en $10^{5} \mathrm{~Pa}$ ainsi que la pression $\mathrm{pz}$ corrigée du poids de la colonne d'eau entre la sonde au moment de l'étalonnage et la position de la sonde pendant l'essai.

En reportant les volumes au bout d'une minute en fonction des pressions $\mathrm{pz}$ sur le graphique situé à droite du tableau précédent, on peut aisément vérifier que le sol est bien mis en pression d'une façon notable par le décollement des points figuratifs correspondants par rapport à la courbe d'étalonnage.

Il est alors possible de passer à la phase suivante qui est la phase de mesures proprement dite.

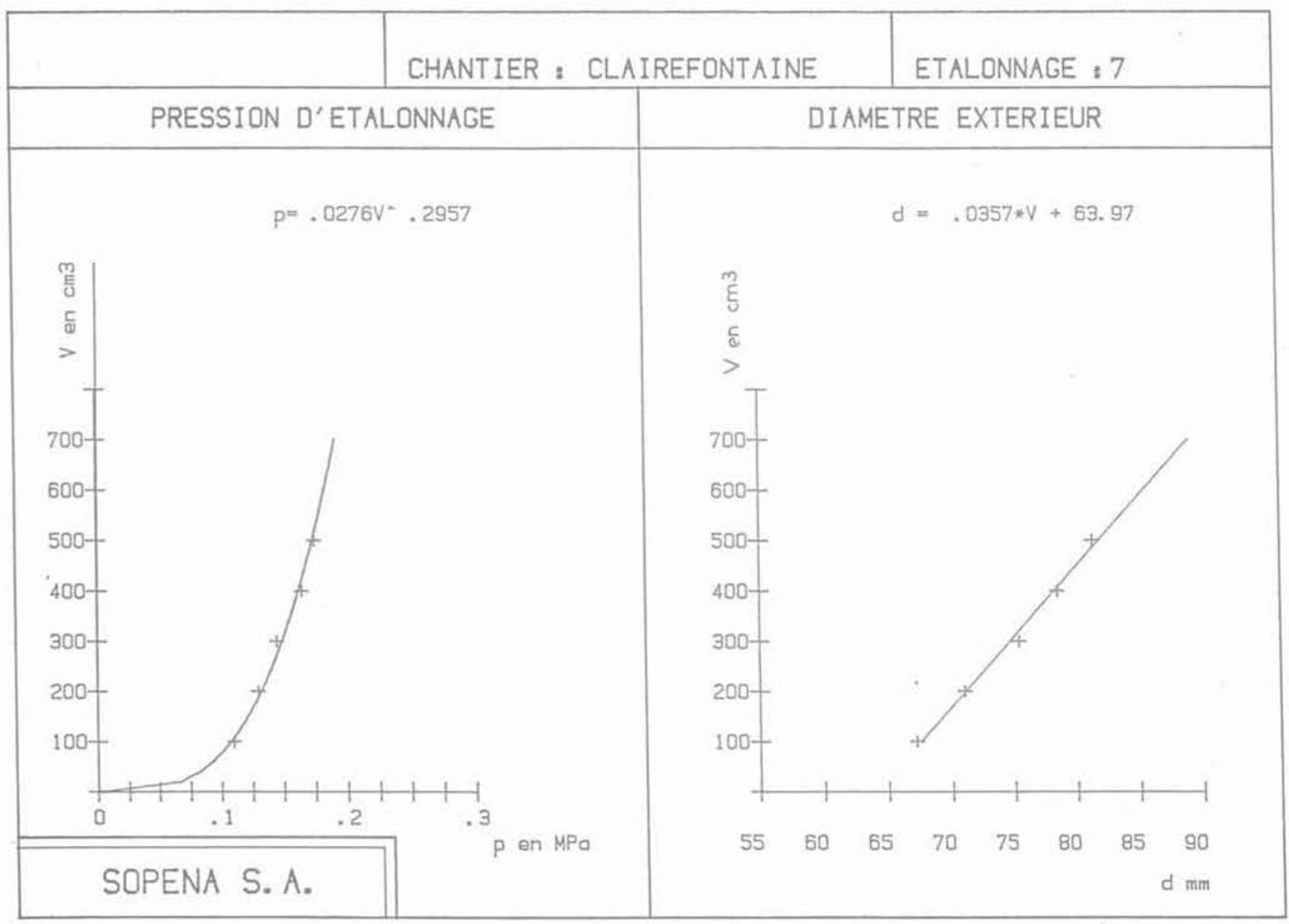

Fig. 5. - Etalonnage de la sonde. 


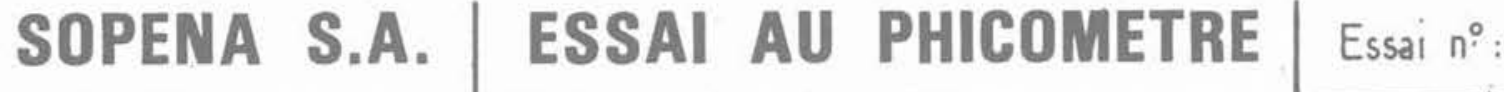

Chantier : Chartzes Sondage : $\$ 4$

Date: $23-12 \cdot 85$

$H_{0}:-0,50$ Sonde $n^{\circ}:-2$, Etalonnage $n^{\circ}:$ Heure: $g^{n} 30$

I mise en contact

\begin{tabular}{|c|c|c|c|c|c|}
\hline Tression & V & $\Delta V$ & Pression & V & $\Delta V$ \\
\hline \multirow{3}{*}{$P_{M 0, \delta}$} & Ho & & \multirow{3}{*}{$\begin{array}{l}P_{M 2,0} \\
P_{Z}{ }_{2,3} \delta\end{array}$} & 170 & \\
\hline & 46 & & & 173 & \\
\hline & 48 & & & 175 & \\
\hline \multirow{3}{*}{$\mathrm{P}_{M} \mathrm{~A}_{0}$} & 110 & & \multirow{3}{*}{$\begin{array}{l}P_{M} \\
P_{Z}\end{array}$} & & \\
\hline & 112 & & & & \\
\hline & 114 & & & & \\
\hline \multirow[t]{3}{*}{$P_{M / 2}$} & 143 & & \multirow{3}{*}{$\begin{array}{l}P_{M} \\
P_{Z}\end{array}$} & & \\
\hline & 145 & & & & \\
\hline & 146 & & & & \\
\hline
\end{tabular}
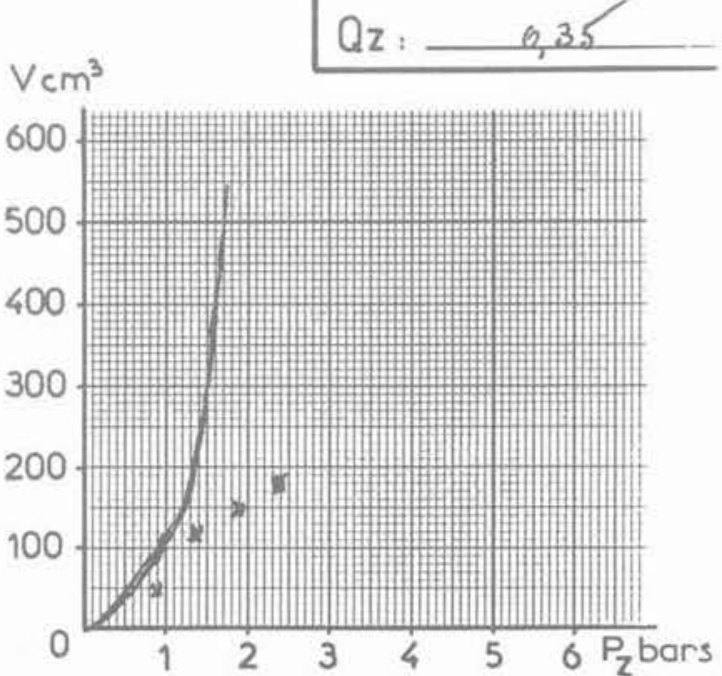

II Paliers de mesure

\begin{tabular}{|c|c|c|c|c|c|c|c|c|c|c|c|}
\hline No & $P_{M}$ & $\Delta \ell$ & T & Polier & $P_{M}$ & $\Delta \ell$ & $T$ & Palier & $P_{M}$ & $\Delta \ell$. & $T$ \\
\hline \multirow{9}{*}{ (1) } & \multirow{9}{*}{2.0} & 1 & 210 & VD & $155^{4} / 27^{\prime}$ & $30^{01} / 273$ & $1^{\prime} / 274$ & VD & 366 & 368 & 370 \\
\hline & & 2 & 320 & \multirow{8}{*}{ (3) } & \multirow{8}{*}{3} & 12 & 660 & \multirow{8}{*}{ (5) } & \multirow{8}{*}{4.5} & 22 & 1200 \\
\hline & & 3 & 380 & & & 13 & 660 & & & 23 & 1290 \\
\hline & & 4 & 390 & & & 14 & 700 & & & $2 / 4$ & $13 \delta_{0}$ \\
\hline & & 5 & 390 & & & 15 & 700 & & & 25 & 1380 \\
\hline & & 6 & 390 & & & 16 & 700 & & & 26 & 1380 \\
\hline & & & & & & & & & & & \\
\hline & & & & & & & & & & & \\
\hline & & VF: & 217 & & & VF: & 296 & & & V.F: & 387 \\
\hline VD & $155 / 234$ & $30^{\circ} / 236$ & 11236 & VD & 308 & 309 & 311 & VD & 405 & 408 & 411 \\
\hline \multirow{8}{*}{ (2) } & \multirow{8}{*}{2,5} & 7 & 510 & \multirow{8}{*}{ (4) } & \multirow{8}{*}{3.5} & 17 & 850 & \multirow{8}{*}{ (6) } & \multirow{8}{*}{5,5} & 27 & 1600 \\
\hline & & 8 & $5 \lambda_{0}$ & & & 18 & 880 & & & 28 & 1730 \\
\hline & & 9 & 530 & & & 19 & 930 & & & 29 & 1800 \\
\hline & & $1_{0}$ & 530 & & & 20 & 940 & & & 30 & 1800 \\
\hline & & 11 & 530 & & & 21 & 940 & & & 31 & 1820 \\
\hline & & & & & & & & & & 32 & 1820 \\
\hline & & & & & & & & & & & \\
\hline & & VF: & 257 & & & $V F:$ & 332 & & & $V F$ & 440 \\
\hline
\end{tabular}


Phase 3 : mesures par paliers successifs :

Réalisation du premier palier :

Le premier palier correspond au dernier de la phase précédente, c'est-à-dire à une pression au manomètre de $200 \mathrm{kPa}$ sur notre exemple.

Pendant toute la réalisation du palier, la pression est maintenue constante au manomètre, c'est-à-dire pour ce palier-ci : $200 \mathrm{kPa}$. On commence ensuite à exercer l'effort d'arrachement à la vitesse de $2 \mathrm{~mm}$ par minute et l'on relève l'effort $T$ sur la cale dynamométrique toutes les 30 secondes. L'essai est poursuivi soit jusqu'à une quasi-stabilisation de l'effort $T$, soit jusqu'à un déplacement maximal de $9 \mathrm{~mm}$ pour ce premier palier. Le volume de la sonde appelé volume final VF est alors lu, soit sur la figure $6: 217 \mathrm{~cm}^{3}$.

Pour la réalisation des paliers suivants, on procède comme suit :

a) Montée à la pression désirée, par exemple $450 \mathrm{kPa}$ pour le $5^{\mathrm{e}}$ palier, mesures des volumes lus au bout de $15 \mathrm{~s}, 30 \mathrm{~s}$ et $1 \mathrm{mn}$ avant de commencer l'arrachement; soit sur la feuille pour le $5^{e}$ palier, respectivement 366,368 et $370 \mathrm{~cm}^{3}$.

D'où obtention d'un premier fluage que nous appellerons le "fluage initial» qui est égal ici à VD $(1 \mathrm{mn})$ - VD $(30 \mathrm{~s})$, c'est-à-dire $2 \mathrm{~cm}^{3}$.

b) Ensuite application de l'effort d'arrachement comme précédemment, avec toujours relevés des efforts $T$ toutes les $30 \mathrm{~s}$ jusqu'à soit une quasistabilisation de l'effort $T$, soit un déplacement maximal de $7 \mathrm{~mm}$.

c) Mesure du volume en fin de palier de cisaillement que l'on appelle le volume final VF, ici VF $=387 \mathrm{~cm}^{3}$.

On peut alors définir un deuxième fluage que nous appellerons fluage de cisaillement qui est égal à $\mathrm{VF}-\mathrm{VD}(1 \mathrm{mn})$, soit ici $17 \mathrm{~cm}^{2}$.

Il est réalisé en général entre cinq et huit paliers de cisaillement. Il a été établi une procédure extrêmement précise pour le choix des incréments de pression entre chaque palier ceci en fonction de la pression limite du sol testé.

Le déplacement vertical total pour la réalisation d'un essai est en général de 30 à $50 \mathrm{~mm}$.

Phase 4 : dépouillement :

Pour chaque palier la contrainte normale $\sigma$ appliquée au sol correspond à la pression corrigée avec les mêmes corrections que pour l'essai pressiométrique. On peut écrire $\sigma=\mathrm{pc}$.

Par ailleurs, la contrainte de cisaillement correspondante $\tau$ est donnée par la formule $\tau=T / S(V)$.

T étant l'effort maximal d'arrachement mobilisé au cours du palier correspondant.

Phase 5 : présentation des résultats :

Les résultats sont présentés sous forme de plusieurs courbes.
La courbe intrinsèque avec les valeurs correspondantes de $\varphi$ i et ci et d'autre part des deux courbes de fluage VD (1 mn) - VD (30 s): fluage initial et VF - VD (1 mn) : fluage de cisaillement.

Ce dernier fluage, nous allons le voir, joue un rôle important dans la compréhension de l'essai.

Une courbe donnant l'évolution du volume en fonction de la pression corrigée pendant tout le déroulement de l'essai est également présentée. Cette courbe ayant moins d'importance n'a pas été reproduite afin de simplifier la lecture des figures.

\section{EXEMPLE DE RÉSULTATS}

La figure 7 montre le résultat d'un essai réalisé dans le sable de Fontainebleau. La figure du haut représente la courbe intrinsèque du sol. La courbe du bas en traits épais représente le fluage de cisaillement.

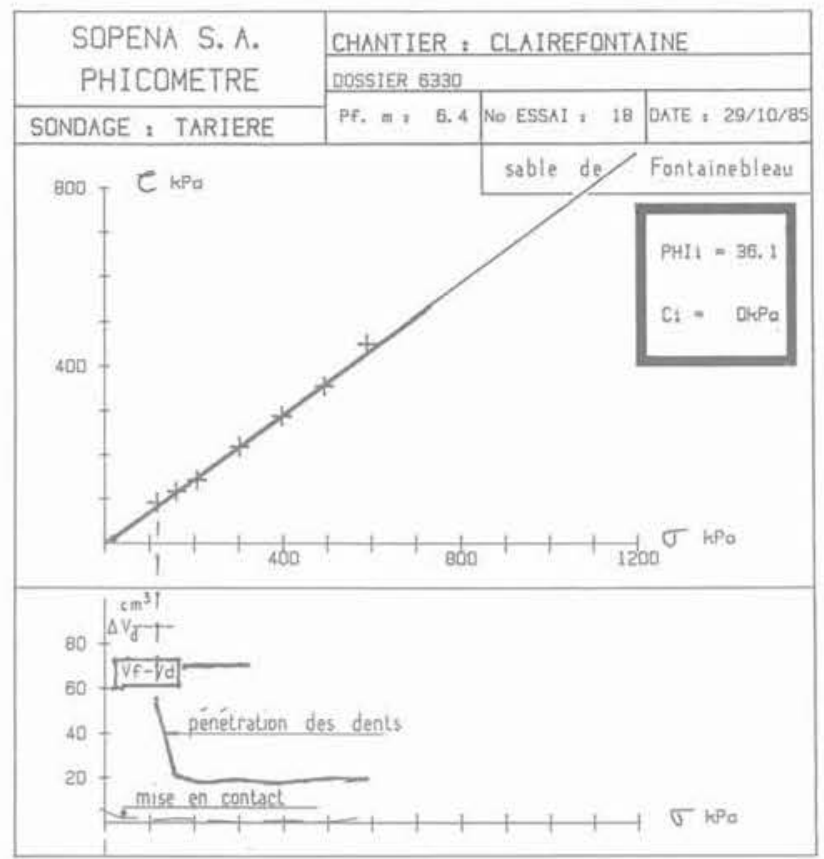

Fig. 7. - Résultat d'un essai sur un sable.

Cet essai appelle deux remarques :

a) Un très important fluage de cisaillement apparaît au cours du premier palier. Ce fluage est attribué à la pénétration complète des dents dans le sol.

b) Il a pu être réalisé sept paliers et les points s'alignent d'une façon assez remarquable, ce qui permet d'ajuster la courbe intrinsèque avec une grande précision.

La figure 8 représente les résultats d'un essai dans un sable argileux. On constate que : 


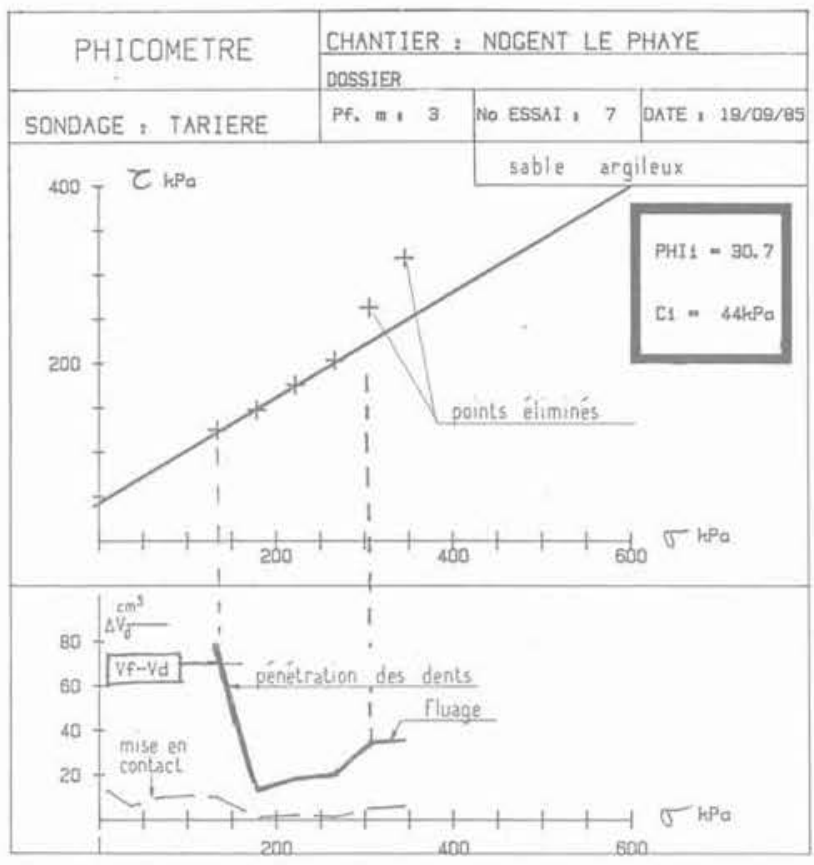

Fig. 8. - Résultat d'un essai sur un sable argileux.

a) Le fluage de cisaillement est encore très important au cours du premier palier, toujours attribué à la pénétration des dents.

b) Au cours des derniers paliers de cisaillement, le fluage de cisaillement redevient relativement important et que consécutivement deux points se situent sensiblement au-dessus de la courbe intrinsèque.

La figure 9 représente les résultats d'un essai dans une argile. On constate :

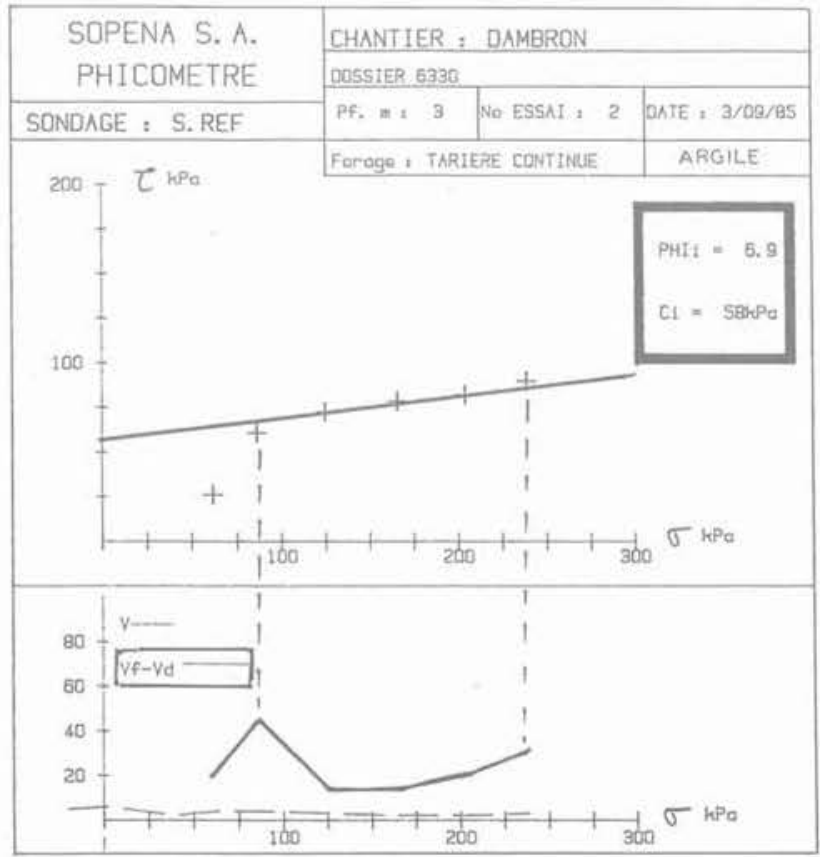

Fig. 9. - Résultat d'un essai sur une argile. a) Une pénétration tardive des dents, le fluage ne s'est effectué que sous le deuxième palier, bien entendu les premières valeurs de $\tau$ sont sous-estimées et sont à éliminer pour l'interprétation.

b) Un début de fluage au dernier palier auquel correspond une contrainte de cisaillement légèrement trop élevée.

\section{ALLURE GÉNÉRALE DE L'ESSAI}

Les exemples précédents n'ont pas été choisis au hasard. L'examen de l'ensemble des essais réalisés à ce jour, nous conduit à décrire l'allure générale de l'essai comme suit :

Si un grand nombre de paliers sont réalisés depuis une contrainte normale faible. jusqu'à des contraintes entrainant un important fluage de cisaillement, on observe (figure 10) :

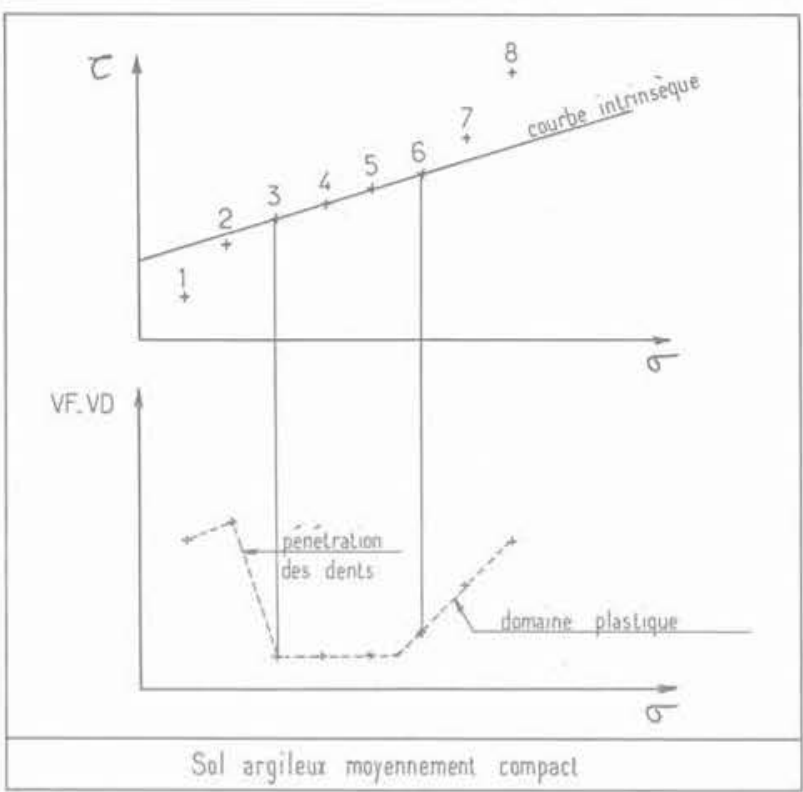

Fig. 10. - Allure des paliers de cisaillement.

1. Que sous les premiers paliers les valeurs de $\tau$ sont sous-estimées.

2. Que sous les derniers paliers, les valeurs sont généralement surestimées dans les sols à comportement argileux. Cependant ceci n'est pas une règle générale et notamment dans les sols à comportement granulaire, les valeurs de $\tau$ sont souvent correctes et bien alignées, même lorsque le fluage de cisaillement devient sensible.

Cette anomalie est attribuée provisoirement à une mise en butée du sol par la partie supérieure de la sonde lorsque son diamètre devient sensiblement plus grand que celui du forage. Ces observations sont très utiles pour la conduite et le dépouillement des essais. 
3. En ce qui concerne le déplacement maximum sous chaque palier de cisaillement, on constate que dans les sols présentant normalement un radoucissement le pic n'est pratiquement jamais observé au cours de l'essai «in situ».

P. HABIB (1984) a montré qu'il existait un effet d'échelle dans la boîte de cisaillement comme dans l'essai triaxial et indique que la progression de la rupture dans une boîte très grande donnerait un effort de résistance moyen qui éliminerait le pic; c'est exactement ce que nous avons observé.

On peut considérer que la résistance mesurée ici est une résistance de palier à ne pas confondre avec la résistance résiduelle qui intervient après des déplacements beaucoup plus importants.

Dans les sols qui présentent de l'écrouissage, on a les mêmes allures de courbe au phicomètre et en laboratoire, et il est, comme en laboratoire, nécessaire de définir conventionnellement la valeur de la résistance à la rupture.

On sait (HABIB 1985) que les déplacements nécessaires pour mobiliser la résistance de pic sont de 3 à $5 \mathrm{~mm}$ tant dans les sables que dans les argiles.

A titre comparatif, il est intéressant de noter que l'allure de la courbe de la mobilisation du frottement latéral entre un pieu et un sol est similaire et que le frottement limite est pratiquement atteint pour un déplacement de 3 à $5 \mathrm{~mm}$ (G. PHILIPPONNAT, 1979, p. 319).

En définitive, un déplacement de $7 \mathrm{~mm}$ nous semble une bonne valeur pour s'assurer que le maximum a été atteint ou dépassé dans le cas des sols rigides ou que la résistance mesurée peut être considérée comme une résistance conventionnelle de palier pour les sols écrouissables.

Par ailleurs, la figure 11 montre l'état de la sonde après un essai dans le sable de Fontainebleau. D'une façon générale, et à l'exception des sols très grossiers, lorsque la sonde est remontée sans trop de heurts, l'espace entre les dents est rempli par les matériaux cisaillés.

\section{ESSAIS COMPARATIFS}

Des essais comparatifs entre le phicomètre et des essais classiques de cisaillement non consolidé rapide en laboratoire ont été réalisés sur une argile marneuse, un sable argileux, et un sable propre.

Afin de ne pas multiplier les paramètres, ils ont tous été effectués dans les conditions suivantes:

- site sans nappe phréatique,

- forages exécutés à la tarière hélicoïdale continue.
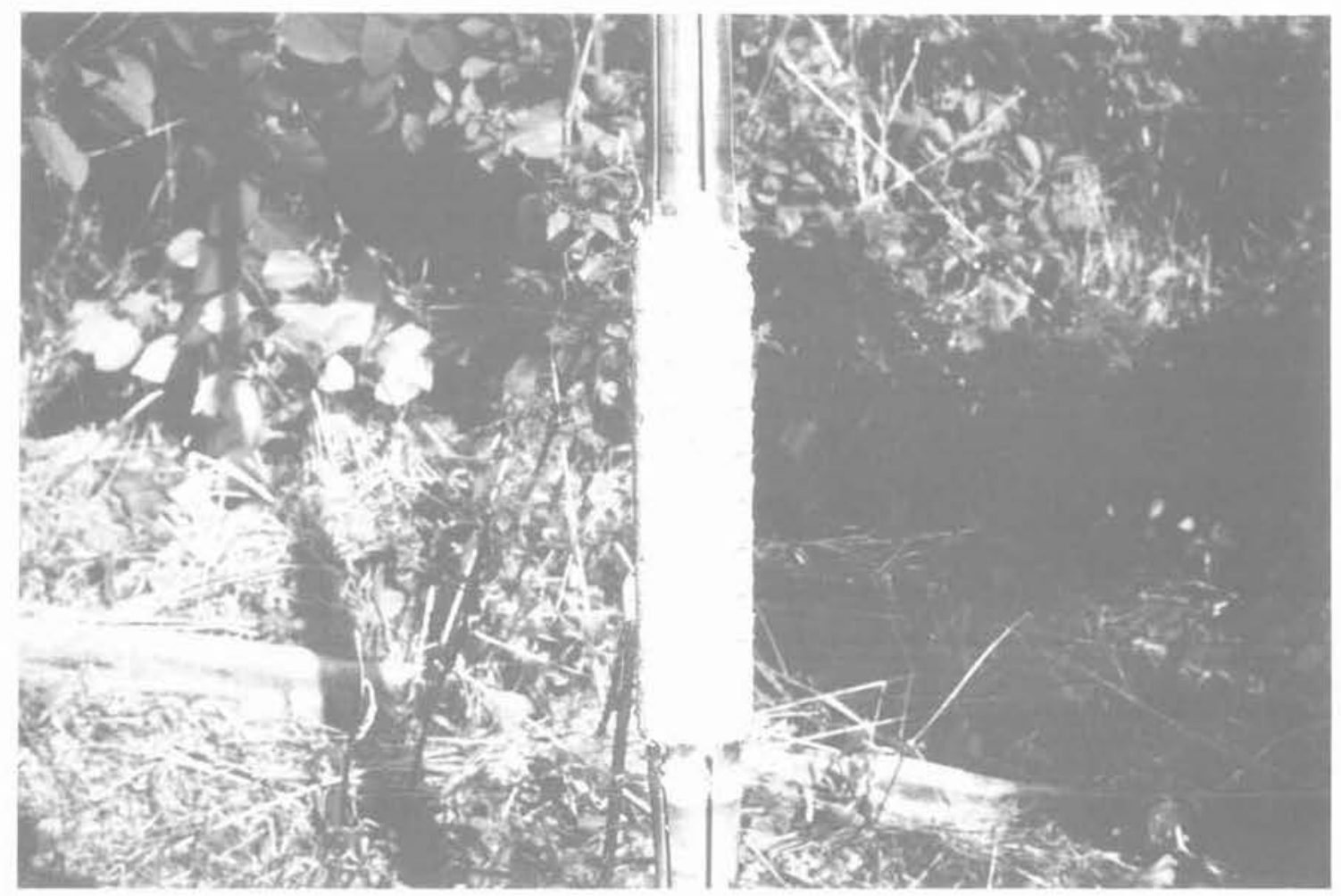

Fig. 11. - Etat de la sonde après essai dans un sable argileux. 


\subsection{Essais comparatifs dans une argile marneuse}

Les essais ont été réalisés à l'emplacement d'un poste de transformation de l'E.D.F. situé à Dambron (28).

Le sol est constitué sur 4 mètres d'épaisseur par une argile marneuse tertiaire très plastique dont les caractéristiques sont les suivantes :

Limites d'Atterberg $: w_{1}=70 \%$ Ip $=40$

Teneur en eau naturelle : $w=29 \%$

Inférieurs à 80 microns : $97 \%$

Pression limite

$$
: \mathrm{pl}=0,6 \text { à } 0,8 \mathrm{MPa}
$$

Des essais comparatifs ont été réalisés à 1,80 et $3 \mathrm{~m}$ de profondeur. Les échantillons intacts pour les essais de cisaillement en laboratoire ont été prélevés par fonçage d'un carottier double à paroi mince.

Le tableau 1 montre les résultats obtenus :

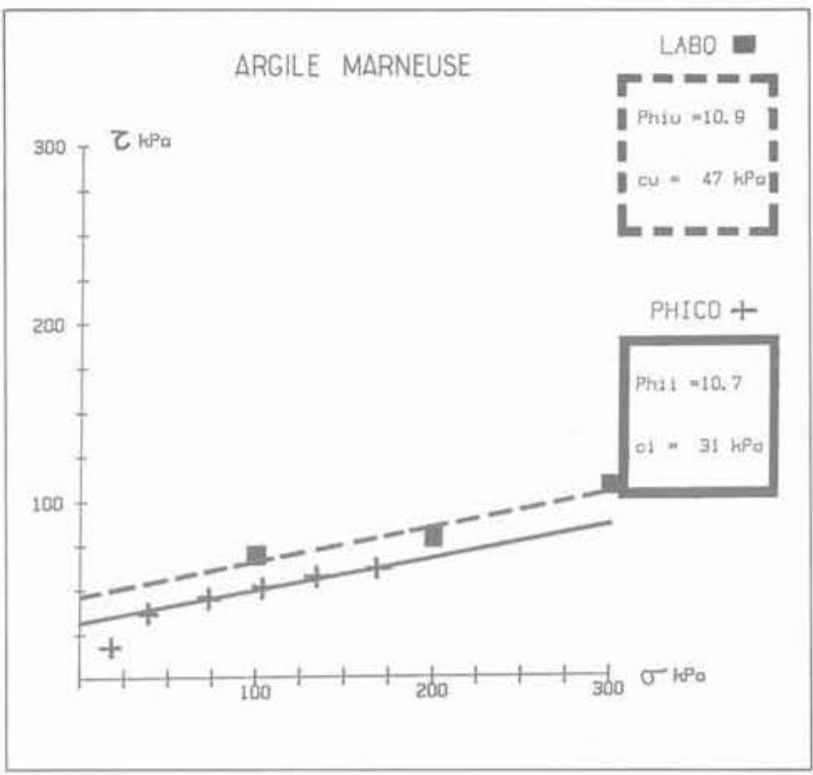

Fig. 12. - Dambron.

Essai à $1,80 \mathrm{~m}$ de profondeur.

Tableau I. - Argile marneuse : essais comparatifs.

\begin{tabular}{|c|c|c|c|c|c|c|}
\hline \multirow{3}{*}{ Profondeur en mètre } & \multicolumn{2}{|c|}{ PHICOMETRE } & \multicolumn{4}{|c|}{ LABORATOIRE } \\
\hline & \multirow{2}{*}{ фi degré } & \multirow{2}{*}{ ci kPa } & \multicolumn{2}{|c|}{ OCTOBRE 1985} & \multicolumn{2}{|c|}{ AVRIL 1985} \\
\hline & & & $\varphi$ u degré & cu kPa & фu degré & $\mathrm{cu} \mathrm{kPa}$ \\
\hline 1,80 & 10,7 & 31 & 10,9 & 47 & 3,1 & 50 \\
\hline 3,00 & $\begin{array}{c}10,0^{*} \\
6,4^{* *}\end{array}$ & $\begin{array}{l}48^{*} \\
59^{* *}\end{array}$ & 14,2 & 47 & 8,3 & 58 \\
\hline
\end{tabular}

* $1^{\text {re }}$ interprétation.

* $2^{\text {n }}$ interprétation.

Dans ce tableau on a reporté les essais de laboratoire réalisés en même temps que les essais au phicomètre en octobre 85 et également les résultats d'essais réalisés en avril 85, au moment de l'étude générale du poste. En ce qui concerne l'essai phicométrique à $3 \mathrm{~m}$ de profondeur, nous avons donné la première interprétation faite au moment des essais, et la deuxième interprétation qui tient compte des commentaires du paragraphe 8.

Une première constatation peut être faite : Pordre de grandeur des résultats est comparable.

Sur les figures 12 et 13, sont reportés les points figuratifs des deux types d'essais in situ et en laboratoire. Il apparait qu'ici, le phicomètre donne une résistance au cisaillement légèrement plus faible que les essais de laboratoire.

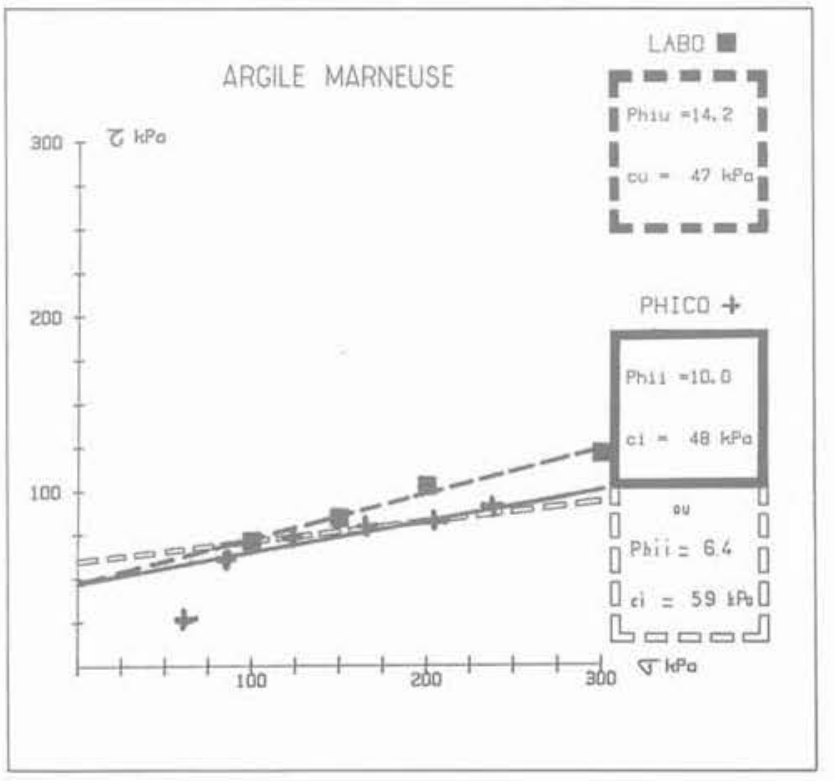

Fig. 13. - Dambron.

Essai à 3,00 m de profondeur. 
Toutefois, il convient de ne pas porter de jugement hâtif. Toute expérimentation sur des sites réels doit être réalisée sur un grand nombre d'essais pour dégager des lois générales car on se heurte à l'hétérogénéité des sites et à la dispersion des essais, et notamment des essais de laboratoire comme nous le verrons plus loin.

\subsection{Essais comparatifs dans des sables argileux}

Ces essais ont été réalisés à Nogent-le-Phaye (28) sur un site où le sol est constitué par des poches résiduelles d'un complexe sablo-argileux de l'Yprésien.

Les essais d'identification ont permis de distinguer deux couches:

- de 0 à $4 \mathrm{~m}$ de profondeur :

- limites d'Atterberg $\quad: w_{1}=25 \% \quad \mathrm{lp}=25$

- teneur en eau naturelle : $w=16 \%$

— inférieurs à 80 microns : $58 \%$

- pression limite moyenne : $\mathrm{pl}=1,4 \mathrm{MPa}$

- de 4 à $6 \mathrm{~m}$ :

- limites d'Atterberg $: w_{1}=22 \% \quad$ lp $=6$

- teneur en eau naturelle

$: w=13 \%$

- inférieurs à 80 microns

: $38 \%$

- pression limite

$: \mathrm{pl}=1,7$ à $3 \mathrm{MPa}$

L'observation des matériaux en cours de forage montre que le sol a un aspect de plus en plus sableux avec la profondeur.

Il a été réalisé quatre essais comparatifs.

Les prélèvements d'échantillons intacts ont été effectués par fonçage d'un carottier double à paroi mince.

Les résultats de ces essais sont rassemblés dans le tableau II.

Tableau II. - Sable argileux : essais comparatifs (novembre 1985).

\begin{tabular}{|c|c|c|c|c|}
\hline \multirow{2}{*}{$\begin{array}{c}\text { Profondeur } \\
\text { en mètre }\end{array}$} & \multicolumn{2}{|c|}{ PHICOMĖTE } & \multicolumn{2}{c|}{ LABORATOIRE } \\
\cline { 2 - 5 } & $\varphi$ i degré & ci kPa & $\varphi$ u degré & cu kPa \\
\hline 1,80 & 33,3 & 68 & 27,6 & 42 \\
3,00 & 30,7 & 44 & 29,7 & 34 \\
4,20 & 32,6 & 29 & 33,1 & 16 \\
5,40 & 36,0 & 0 & $(31)$ & 0 \\
\hline
\end{tabular}

L'examen de ce tableau appelle les remarques suivantes :

a) L'essai en laboratoire à $5,40 \mathrm{~m}$ de profondeur donne un angle de frottement interne anormalement faible pour un sol aussi compact : $31^{\circ}$.

b) Cette fois-ci, les caractéristiques mesurées in situ, $\varphi \mathrm{i}$ et ci sont plutôt supérieures à celles mesurées en laboratoire.

c) Sur les trois premiers essais, les valeurs moyennes de l'angle de frottement interne sont les suivantes:

Phicomètre $\varphi$ i moyen $=32,2^{\circ}$

Laboratoire $\varphi \mathrm{u}$ moyen $=30,1^{\circ}$.

d) Bien que les cohésions in situ soient supérieures aux cohésions mesurées en laboratoire, il est très intéressant de remarquer que l'on observe la même décroissance de la cohésion avec la profondeur in situ et en laboratoire.

Les figures 14 à 17 montrent les résultats comparatifs entre les essais réalisés à chaque profondeur.

Il est intéressant de noter que les essais in situ ont dans l'ensemble été réalisés sans difficultés avec des contraintes normales atteignant $600 \mathrm{kPa}$.

En définitive, pour cette série d'essais, on peut encore dire que le phicomètre donne au moins un ordre de grandeur satisfaisant, cette fois-ci par excès, des caractéristiques apparentes $\varphi \mathrm{u}$ et cu.

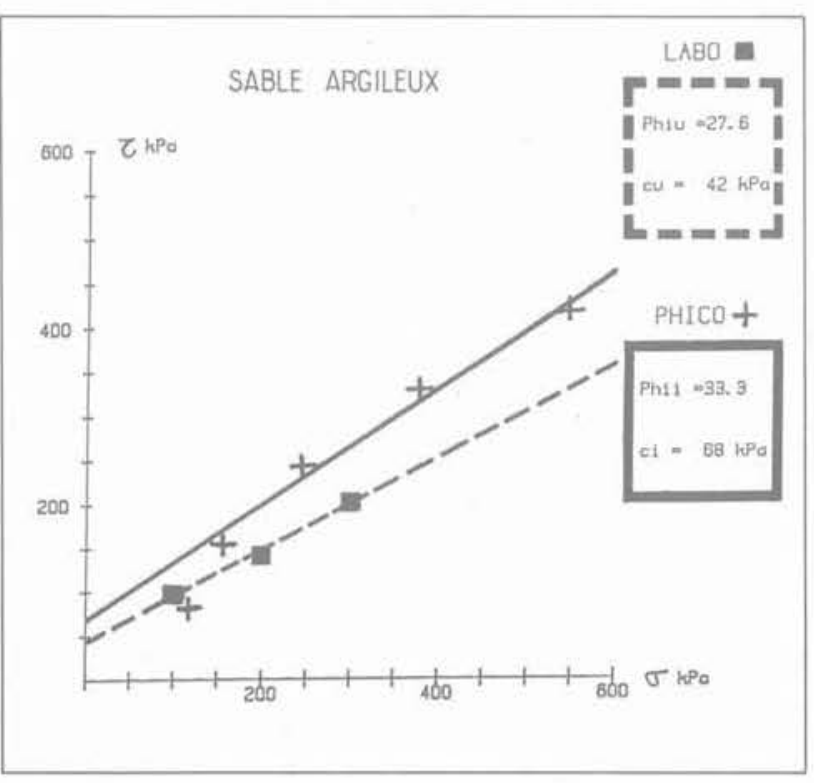

Fig. 14. - Nogent-le-Phaye. Essai à 1,80 $\mathrm{m}$ de profondeur. 


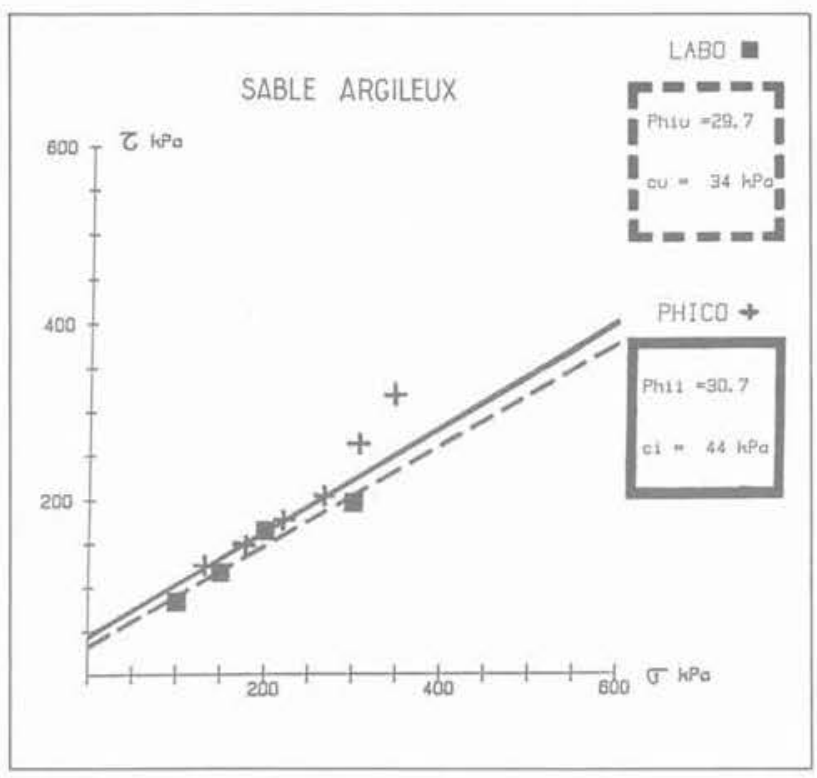

Fig. 15. - Nogent-le-Phaye.

Essai à $3,00 \mathrm{~m}$ de profondeur.

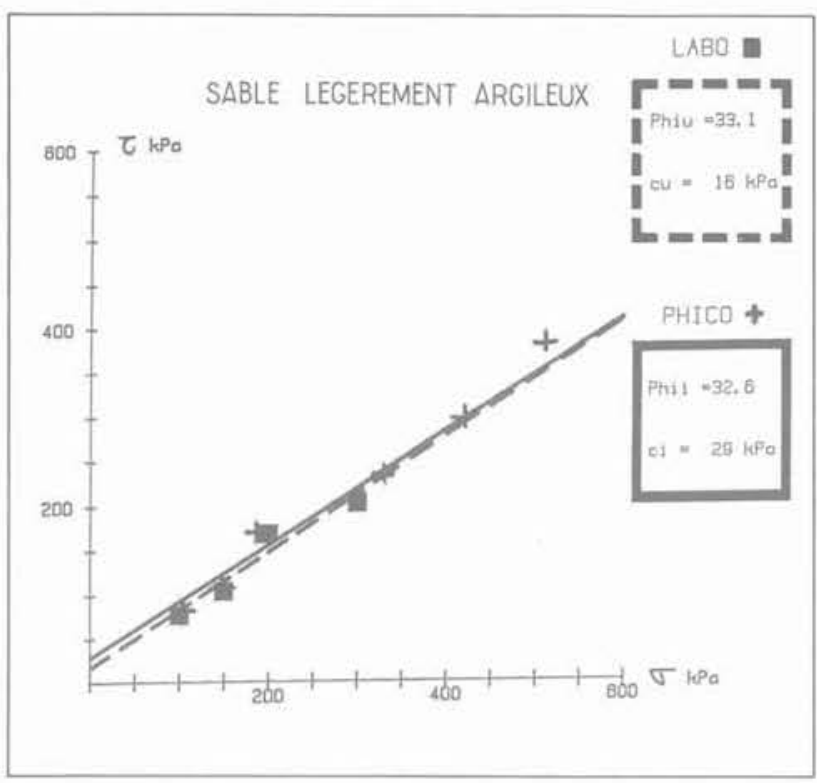

Fig. 16. - Nogent-le-Phaye.

Essai à 4,20 $m$ de profondeur.

\subsection{Essais comparatifs dans le sable propre}

Les essais ont été réalisés dans le terrain de l'Institut National du Football à Clairefontaine (78). Le sol est constitué sur une très grande épaisseur par du sable de Fontainebleau.

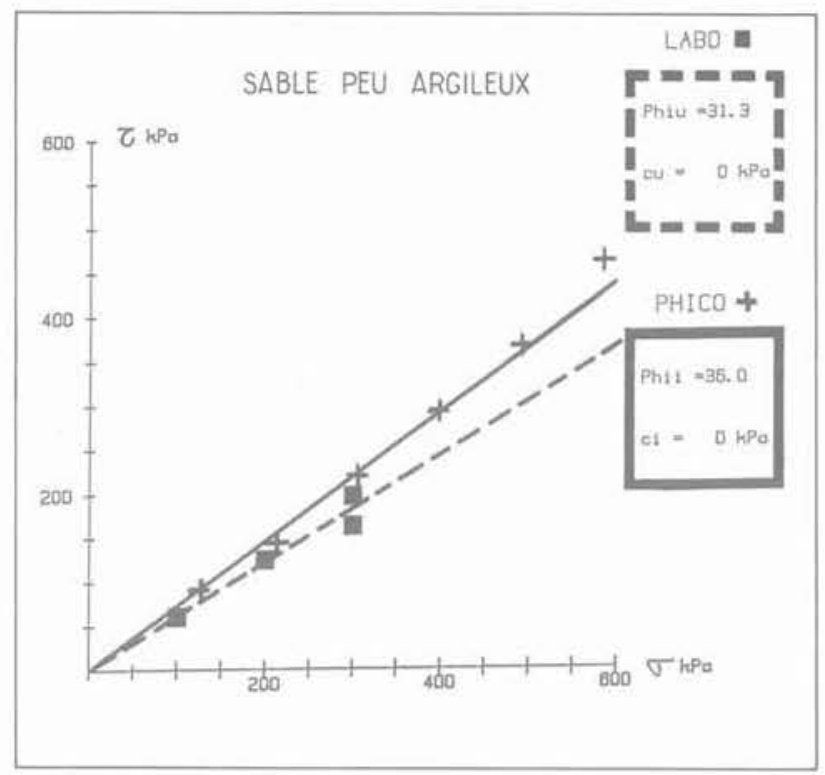

Fig. 17. - Nogent-le-Phaye.

Essai à $5,40 \mathrm{~m}$ de profondeur.

A l'emplacement retenu pour les essais, le sable est lâche et pollué jusqu'à $2,50 \mathrm{~m}$ de profondeur; audelà, il devient propre et compact. Ces sables possèdent les caractéristiques suivantes :

- de 0 à $2,50 \mathrm{~m}$ de profondeur :

- sol non plastique,

- teneur en eau naturelle: $w=4 \%$,

- inférieurs à 80 microns : $21 \%$,

- pression limite $\mathrm{pl}=0,7 \mathrm{MPa}$,

- au-delà de $2,50 \mathrm{~m}$ de profondeur :

- sol non plastique,

- teneur en eau naturelle: $w=4 \%$,

- inférieurs à $0.5 \mathrm{~mm}=100 \%$.

- inférieurs à 80 microns : $1 \%$,

- pression limite $\mathrm{pl}=1,2$ à $2,7 \mathrm{MPa}$.

Il a été réalisé cinq essais depuis $1,80 \mathrm{~m}$ jusqu'à $6,40 \mathrm{~m}$ de profondeur. Les résultats comparatifs obtenus sont donnés dans le tableau III.

Un fait très important est à noter : seul le premier prélèvement a été effectué par fonçage toujours à l'aide d'un carottier double à paroi mince. Pour les autres prélèvements, la sondeuse utilisée étant trop légère eu égard à la compacité du sable, les carottiers ont été battus.

On constate que si logiquement, le phicomètre donne un angle de frottement de $34^{\circ}$ pour le sable lâche et voisin de $36^{\circ}$ en profondeur, un résultat inverse est obtenu en laboratoire. 
Tableau III. - Sable propre : essais comparatifs (novembre 1985).

\begin{tabular}{|c|c|c|c|c|c|c|}
\hline & \multirow{2}{*}{$\begin{array}{l}\text { Profondeur } \\
\text { en mètre }\end{array}$} & \multicolumn{2}{|c|}{ PHICOMETRE } & \multicolumn{3}{|c|}{ LABORATOIRE } \\
\hline & & фi degré & ci $\mathrm{kPa}$ & Prélèvement & qu degré & $\mathrm{cu} \mathrm{kPa}$ \\
\hline Sable lâche & 1,80 & 34,1 & 0 & $\mathrm{~F}$ & 38,0 & 0 \\
\hline Sable compact & 3,00 & 36,3 & 0 & B & 32,0 & 0 \\
\hline « & 3,80 & 35,8 & 0 & B & 33,0 & 0 \\
\hline " & 5,40 & 35,3 & 0 & B & 33,5 & 0 \\
\hline " & 6,40 & 36,1 & 0 & B & 32,0 & 0 \\
\hline
\end{tabular}

$\mathrm{F}=$ prélèvement par fonçage

$\mathrm{B}=$ prélèvement par carottier battu

Ceci nous a conduit à analyser l'influence du mode de prélèvement.

Au cours de l'étude générale du site, un assez grand nombre d'essais ont été effectués sur le sable de Fontainebleau.

Disposant à l'époque d'une sondeuse lourde, tous les échantillons ont été foncés. L'analyse des valeurs de l'angle de frottement obtenues en fonction du mode de prélèvement conduit aux résultats suivants :

- carottier battu, quatre essais :

(il s'agit des quatre essais réalisés à côté des phicomètres entre $3 \mathrm{~m}$ et $6,40 \mathrm{~m}$ de profondeur)

$-\varphi$ moyen : $32,6^{\circ}$,

$-\phi$ minimum : $32^{\circ}$,

- $\varphi$ maximum : $33,5^{\circ}$;

- carottier foncé, cinq essais :

$-\varphi$ moyen $: 38,4^{\circ}$,

$-\varphi$ minimum : $33^{\circ}$,

$-\varphi$ maximum : $40^{\circ}$

La lecture de ces valeurs montre que les deux populations de résultats sont différentes.

Ceci nous conduit à comparer les résultats non pas un par un, mais en superposant sur un même graphique les différents points figuratifs des essais de meme type effectués dans le sable propre et compact. Du même coup, nous nous sommes également intéressés à la fidélité des résultats.

La figure 18 montre les résultats des quatre essais au phicomètre effectués entre $3 \mathrm{~m}$ et $6,40 \mathrm{~m}$ de profondeur. La dispersion des points figuratifs est extrêmement faible; le coefficient de corrélation $\mathrm{r}=0,996$. La fidélité de l'essai est donc excellente.
Les figures 19 et 20 montrent les résultats des essais de cisaillement non consolidés rapides réalisés respectivement sur les échantillons prélevés par battage et par fonçage. On constate d'une part une dispersion des points figuratifs qui est sans comparaison avec la précédente, et d'autre part une différence énorme entre l'estimation de l'angle de frottement interne donné par ces deux séries d'essais.

Sur la figure 21, les points figuratifs de l'ensemble des essais de cisaillement consolidés lents réalisés sur ce site ont été reportés.

L'angle de frottement interne $\varphi^{\prime}$ est de $37^{\circ}$ en moyenne, mais la dispersion est toujours importante.

Enfin sur la figure 22, les meilleurs ajustements des différentes séries d'essais ont été reportés :

\section{SABLE DE FONTAINEBLEAU}

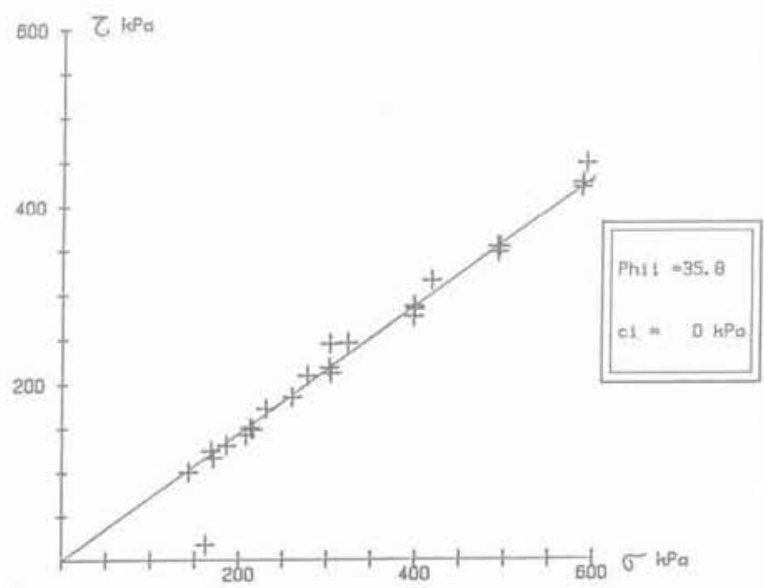

Fig. 18. - Phicomètre. Résultats des quatre essais entre 3,00 et $6,40 \mathrm{~m}$ de profondeur. 
Le carottier foncé fournit les valeurs les plus élevées de l'angle $\varphi \mathrm{u}$.

Le carottier battu donne des valeurs anormalement faibles :

Les essais consolidés lents conduisent à une valeur moyenne de $\varphi^{\prime}=37^{\circ}$.

Les essais phicométriques conduisent à une valeur moyenne de $\varphi_{1}=35,8^{\circ}$, soit pratiquement $36^{\circ}$.

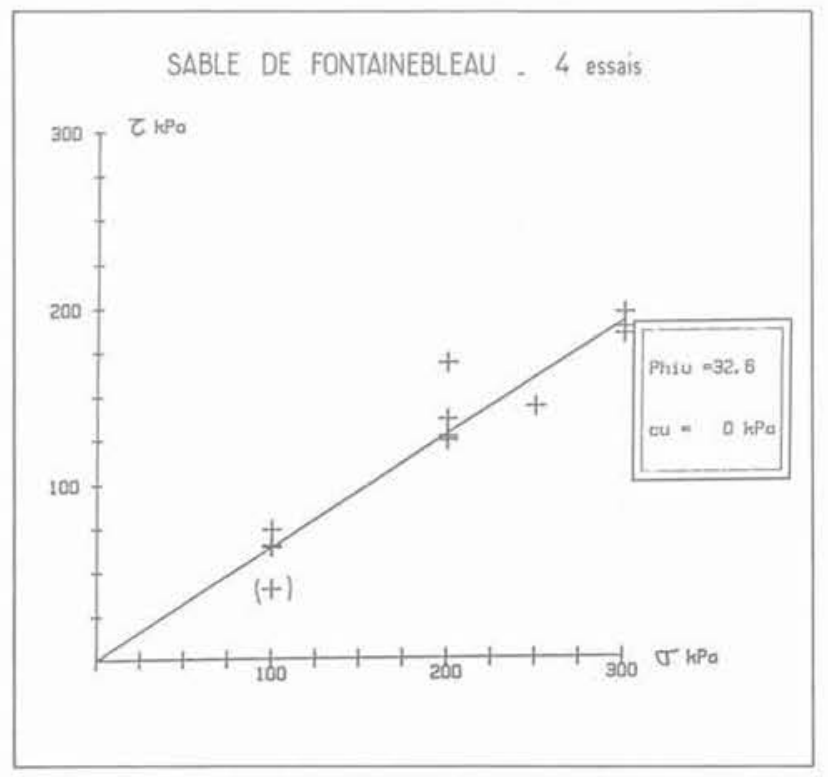

Fig. 19. - Cisaillements non consolidés rapides sur échantillons prélevés par battage.

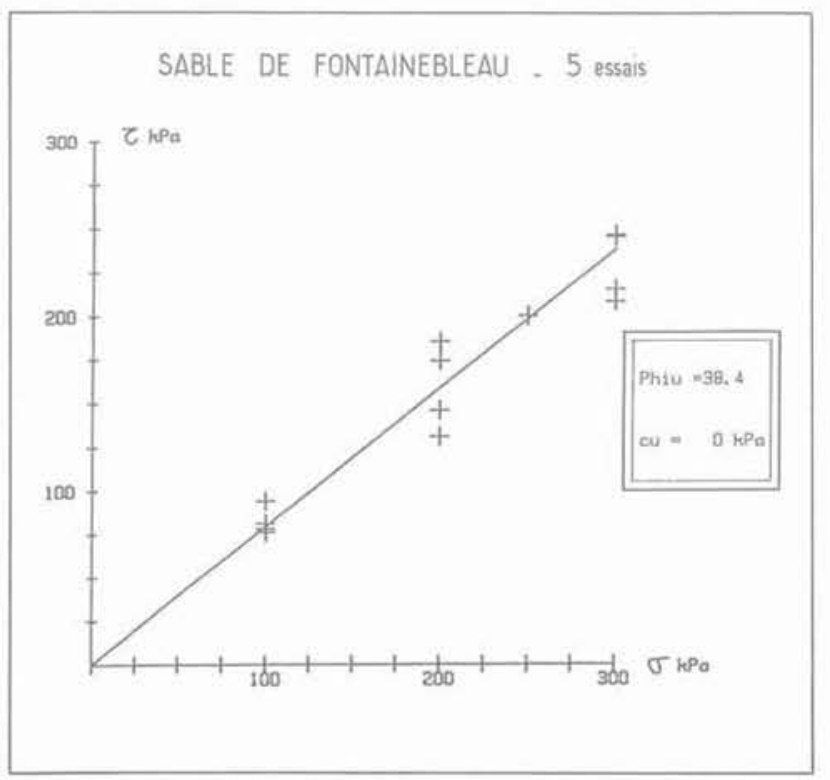

Fig. 20. - Cisaillements non consolidés rapides sur échantillons vérinés.
En définitive, il apparaît sur la base de cette série d'essais :

1. Que le phicomètre donne ici une bonne approximation de l'angle de frottement interne du sable propre et peu humide.

2. Que le phicomètre conduit à une dispersion très faible et donc que l'essai présente une excellente fidélité.

3. Que dans ce type de sol un prélèvement réellement intact est pour le moins difficile à réaliser.

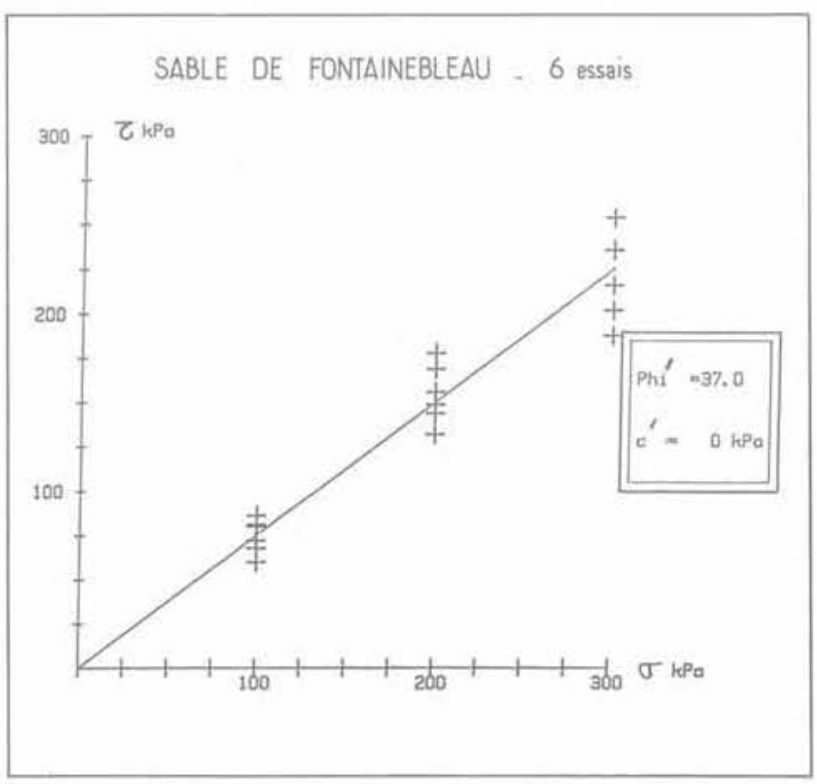

Fig. 21. - Cisaillements consolidés lents sur échantillons vérinés.

\section{SABLE DE FONTAINEBLEAU}

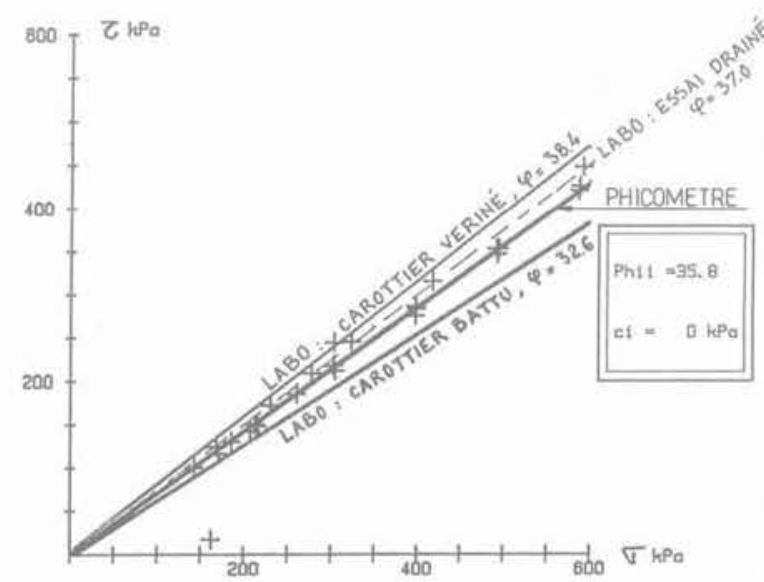

Fig. 22. - Comparaison des droites intrinsèques selon le type d'essai. 


\section{ESSAIS SUR UN MATÉRIAU IMPRÉLEVABLE}

Afin de montrer les perspectives encore mal connues qu'offre le phicomètre, nous allons présenter maintenant les résultats obtenus sur un tout-venant marnocalcaire compacté $0 / 150 \mathrm{~mm}$.

Les essais ont été réalisés dans les remblais qui bordent un silo linéaire situé à Avord dans le Cher. Ces remblais ont une hauteur de $4 \mathrm{~m}$, ils sont constitués par un tout-venant marno-calcaire dont la courbe granulométrique est présentée sur la figure 23.

La figure 24 permet de se faire une idée de la nature du matériau. Ces remblais ont été compactés très énergiquement, et les pressions limites mesurées au pressiomètre sont voisines de $2 \mathrm{MPa}$.

Les forages ont été réalisés en roto-percussion à l'air. Deux essais phicométriques ont été effectués à $1,80 \mathrm{~m}$ et $3 \mathrm{~m}$ de profondeur. Ces essais ont permis de définir les caractéristiques mécaniques données dans le tableau IV.

La figure 25 montre le résultat de l'essai effectué à $3 \mathrm{~m}$ de profondeur. L'essai à $1,80 \mathrm{~m}$ a une allure similaire.
Tableau IV. - Tout-venant marno-calcaire 0/150 mm. Matériau imprélevable: essais d'octobre 1985.

\begin{tabular}{|c|c|c|}
\hline $\begin{array}{c}\text { Profondeur } \\
\text { en mètre }\end{array}$ & pi degré & ci kPa \\
\hline 1,80 & 44,3 & 8 \\
3,00 & 39,2 & 49 \\
\hline Moyenne & 41,7 & 28 \\
\hline
\end{tabular}

On constate :

1. L'excellent alignement des points malgré le fluage qui s'est développé sur les derniers paliers de cisaillement.

2. L'échelle : les essais ont été poussés jusqu'à des pressions normales de l'ordre de $500 \mathrm{kPa}$.

3. Sur la figure 26 , l'ensemble des points figuratifs est reporté pour les deux essais.

La droite de régression correspondante conduit aux valeurs suivantes :

$$
\varphi \mathrm{i}=41,4^{\circ} \text { et } \mathrm{ci}=30 \mathrm{kPa} .
$$

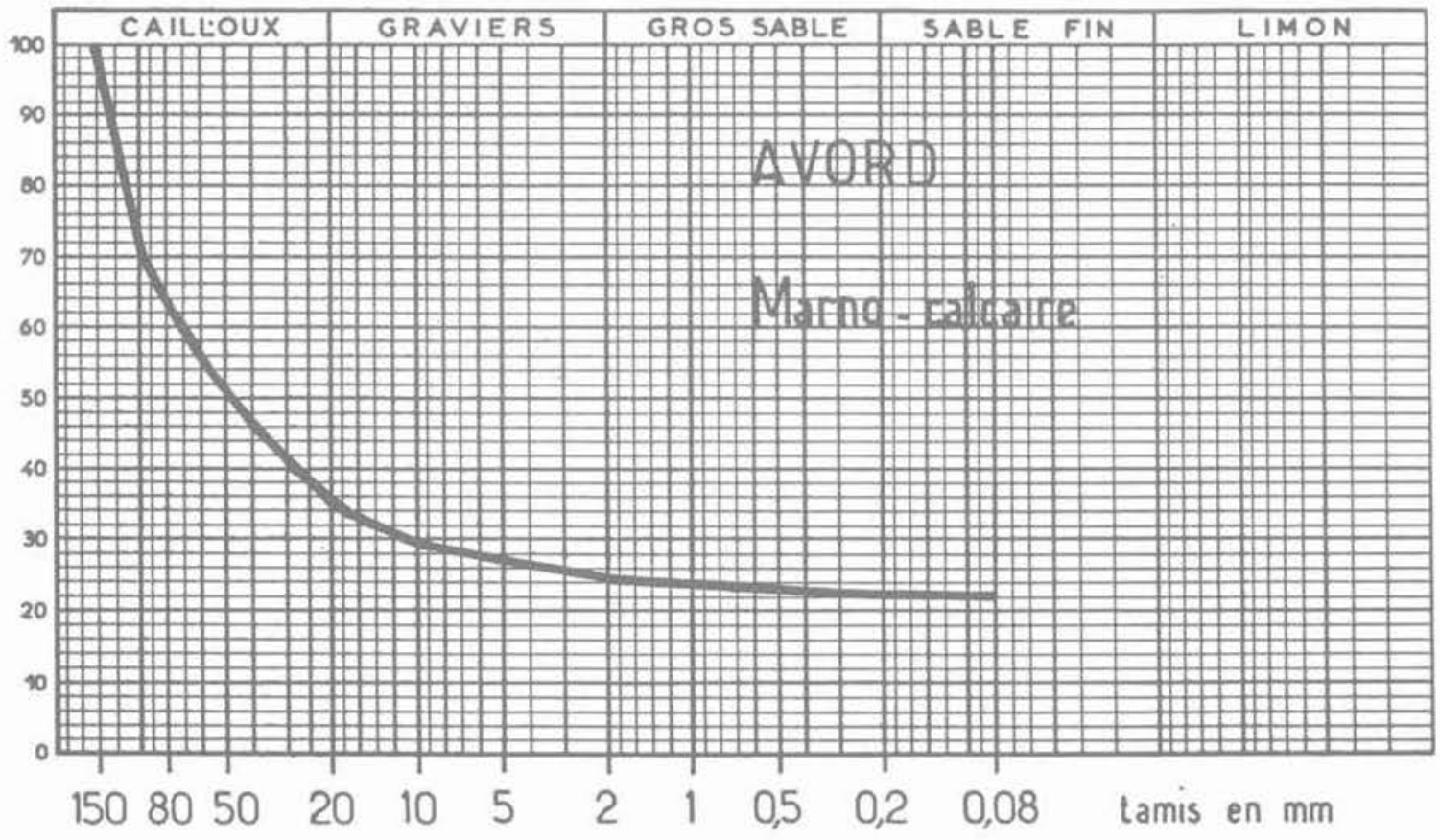

Fig. 23. - Courbe granulométrique du Tout-venant $0 / 150 \mathrm{~mm}$. 


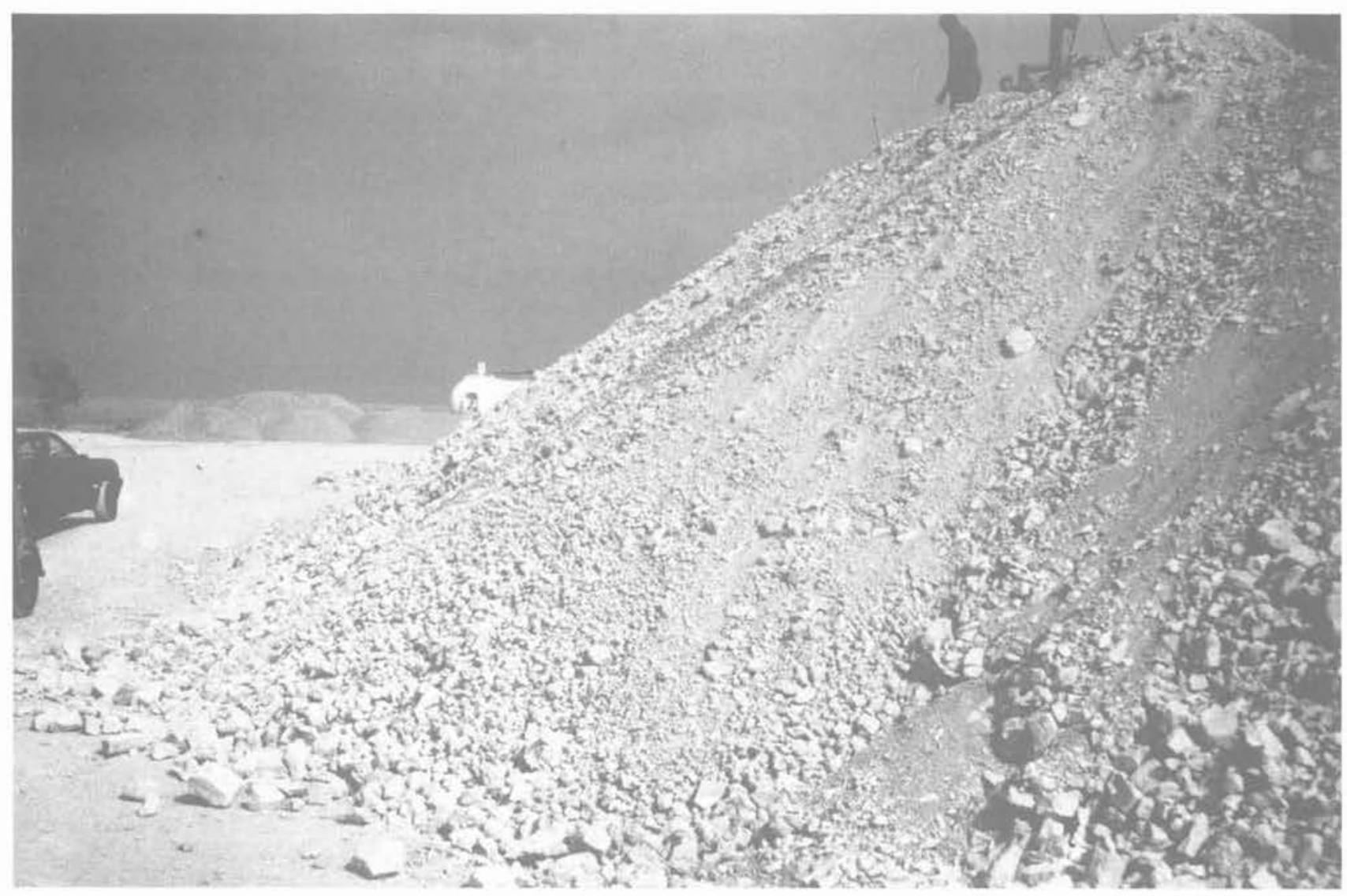

Fig. 24. - Aspect du talus en remblai.

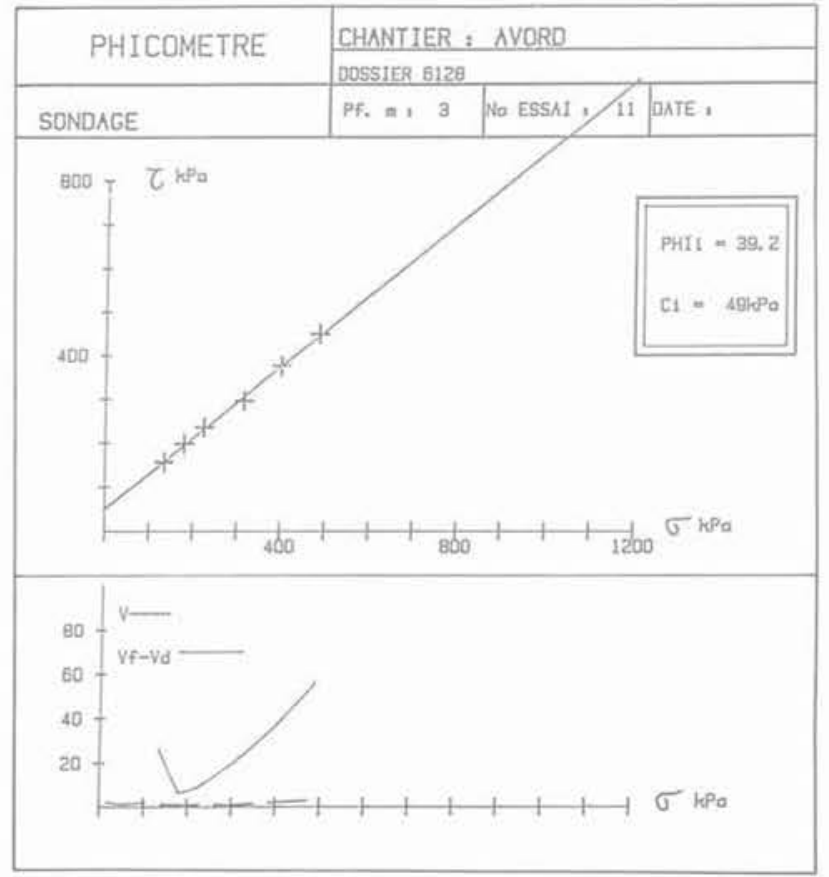

Fig. 25. - Tout-venant $0 / 150 \mathrm{~mm}$. Essai à $3,00 \mathrm{~m}$ de profondeur.

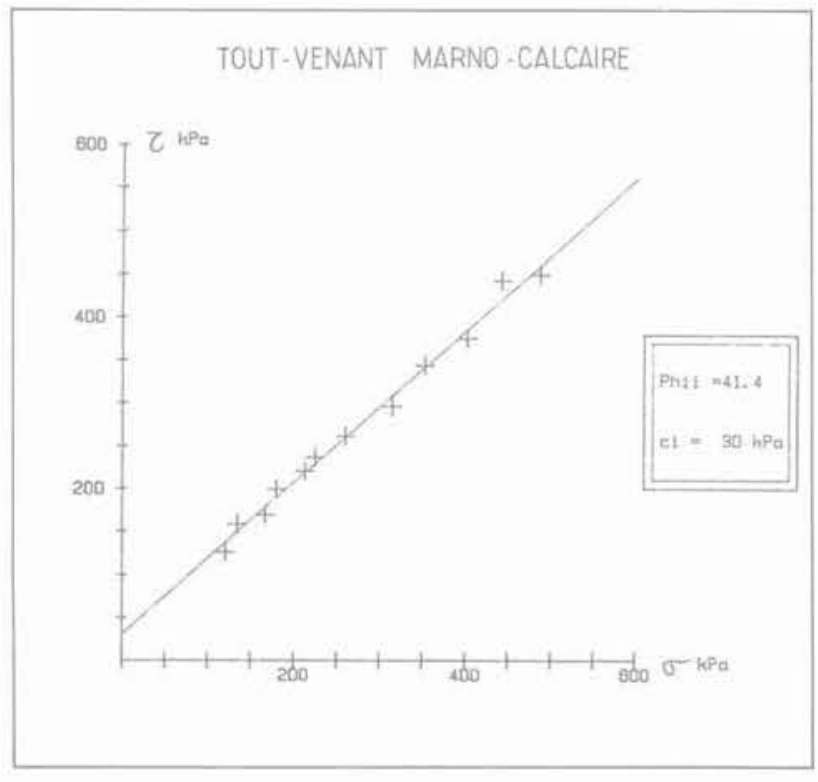

Fig. 26. - Avord. Ensemble des deux essais. 
Bien entendu aucune comparaison avec des essais courants en laboratoire n'est possible mais ces valeurs ne semblent pas absurdes. Elles conduisent à un coefficient de sécurité supérieur à 2 vis-à-vis de la stabilité des talus des remblais du silo.

4. Là encore, la dispersion est très faible, surtout si l'on considère la nature du matériau.

5. L'approche consistant à reporter les points figuratifs de plusieurs essais réalisés dans un massif de sol de même nature semble intéressante pour dégager les valeurs les plus probables de $\varphi \mathrm{i}$ et $\mathrm{ci}$.

\section{CONCLUSIONS}

Cette série d'essais a permis de s'assurer qu'un certain nombre des objectifs que nous nous étions fixés (voir § 2) était déjà atteint.

Simplicité, et coût de l'essai : la durée de l'essai est d'une heure environ, y compris l'introduction de la sonde et le démontage du matériel, le coût d'un tel essai correspond au maximum à celui d'un essai de cisaillement non consolidé rapide en laboratoire, sans compter le prélèvement d'échantillons et le transport dans ce dernier cas.

La robustesse du matériel s'est révélée excellente.

Le diamètre du forage permet d'alterner dans un même forage des essais pressiométriques et des essais phicométriques, d'où la possibilité de mesurer les caractéristiques E, pl, ci et $\varphi$ i.

Ceci peut se révéler particulièrement intéressant par exemple pour le dimensionnement d'une paroi de soutènement par la méthode élasto-plastique.

Le mode opératoire étant bien défini, il est facile de former un opérateur connaissant déjà l'essai pressiométrique.

D'autre part, les résultats actuels permettent déjà d'affirmer que :

- lordre de grandeur des caractéristiques apparentes mesurées est correct,

- que les essais sont réalisables dans des sols grossiers, ce qui permet d'obtenir des caractéristiques mécaniques de sols tels que les alluvions grossières, les tout-venants, l'argile à silex, les remblais divers, etc.,

- la fidélité de l'essai apparaît comme tout à fait prometteuse,

- en ce qui concerne les perspectives d'utilisation, il convient de préciser que plus d'une centaine d'essais sont actuellement en cours notamment pour l'E.D.F. et la S.N.C.F.

Les buts de cette nouvelle série d'essais, en dehors de l'application directe aux projets dans le cadre desquels ils sont réalisés, sont :

- l'analyse statistique comparative avec les essais de laboratoire sur les sols prélevables,

- l'étude de l'influence des moyens de forage sur les résultats d'essais notamment en présence de la nappe phréatique,

- le rassemblement de valeurs des caractéristiques apparentes in situ dans des sols imprélevables tels que les marno-calcaires, l'argile à silex, la craie et les roches altérées.

\section{BIBLIOGRAPHIE}

J. COSTE et G. SANGLERAT (1975), Cours de mécanique des sols, tome II, p. 307, 2e édition, Dunod.

G. MAZIER (1971), Les essais * in situ " en rocher et dans les sols meubles, Session d'Automne 1971. Société Suisse de Mécanique des sols et des roches, Édition Berne.

G. FILLIAT (1981), La pratique des sols et fondations, p. 237 à 240, Edition du Moniteur.

C.P. WROTH (1984), The interpretation of in situ soil tests, Géotechnique, vol. XXXIV, number 4 , p. 449 à 489 .

P. HABIB (1984), Les surfaces de glissements en mécanique des sols, Revue Française de Géotechnique, $n^{\circ} 27$.

P. HABIB (1984), Effet d'échelle et surface de glissement, Revue Française de Géotechnique, n 31.

G. PHILIPPONNAT (1979), Fondations et ouvrages en terre, Edition Eyrolles. 